\title{
Botanische Malerei am Beispiel eines Granatapfels (Punica granatum)
}

Interview mit der Künstlerin Verena Redmann von Christiane Hoegermann

Es ist im Palmengarten bereits mehrjährige Tradition, dass The Society of Botanical Artists aus London alle zwei Jahre in einer Ausstellung Pflanzendarstellungen zu einem bestimmten Thema zeigt. Die Gesellschaft besteht seit 1985 und bietet Künstlern, die sich der Tradition der botanischen Malerei seit dem 16. Jahrhundert widmen, ein Forum.

Auch Verena Redmann aus Nottuln im Münsterland gehört zu der kleinen, aber feinen Gruppe von Botanical Artists. Sie zeichnet mit Farbstiften diverse Pflanzen und deren filigrane Details, die dem Auge des Betrachters gewöhnlich verborgen bleiben. Sie hält sich an die Devise „Das Wesentliche ist für die Augen unsichtbar, man sieht nur mit dem Herzen gut." (nach „Der kleine Prinz" von Antoine DE SAINT-EXUpéry)

Ein Gespräch mit Verena Redmann zeigt, wie sie zur botanischen Zeichnerin geworden ist.

Gab es ein Schlüsselerlebnis, das Sie zur filigranen Darstellung von botanischen Objekten angeregt hat? Wenn ja, welches?

Das Schlüsselerlebnis, das mir die Augen für botanische Kunst öffnete, war ein Artikel in der Zeitschrift „Architektur und Wohnen“ über Shirley Sherwood, eine englische Sammlerin botanischer Kunst. Dort wurde über zeitgenössische botanische Kunst berichtet und Abbildungen von Alpenveilchen, Tulpen, Dschungelpflanzen und Kohlrabi gezeigt. Anhand der abgebildeten Kunstwerke konnte man auch sehen, wie vielseitig Botanik ist und dass Gemüse es durchaus wert ist, gezeichnet zu werden. Dies ist besonders in unserer Zeit wichtig, da viele unserer Kulturpflanzen zu verschwinden drohen oder immer unbekannter werden. Da wusste ich, womit ich mich in Zukunft beschäftigen und was ich zeichnen würde.

Hatten Sie schon in Ihrer Kindheit und Schulzeit eine Begabung für das Zeichnen von Natur- bzw. Realobjekten? Wie äußerte sich diese gegebenenfalls?

Mein Interesse an Botanik zeigte sich schon in meiner Kindheit. Wir fuhren in den Ferien immer nach Österreich. In allen Hotels, Gasthöfen und Liftstationen hingen Plakate, auf denen wunderschöne Pflanzenaquarelle auf geschützte Alpenpflanzen aufmerksam machten. Während nun die anderen Kinder draußen spielten, stand ich fasziniert vor diesen kleinen Kunstwerken. Ich habe heute noch eine Naturtafel mit geschützten Pflanzen in meinem Studio. Und gezeichnet habe ich schon immer.

Welche Bedeutung haben insbesondere alte Obst- und Gemüsesorten für Sie persönlich und bei der Auswahl Ihrer Objekte?

Die alten Obst- bzw. Gemüsesorten haben sehr individuelle Formen und Farben. Man denke nur an das wunderbare Blau der Stangenbohne ,Blauhilde', die beim Kochen das Blau leider verliert und grün wie jede andere Bohne wird. Diese und auch die Ochsenherz-Tomate sind alte Gemüsesorten. Die vielen alten Sorten waren an verschiedene regionale Bodenverhältnisse oder Klimabedingungen angepasst. Es wurden dementsprechend Sorten gepflanzt, die z.B. trockene Sommer sowie nasse Sommer aushalten konnten. So war gewährleistet, dass man auf alle Fälle ernten konnte und nicht hungern musste. Durch eine neue geplante Saatgutverordnung ist diese Vielfalt in Gefahr.

Gemüse kennt man heute hauptsächlich zerkleinert in einer Tiefkühltüte. So verlieren wir den Bezug zu unseren Lebensmitteln. Wir sollten zurückgehen zu den Wurzeln einer sinnlichen Wahrnehmung unserer Lebensmittel und deren Wertschätzung, die Schönheit des Gemüses oder Obstes, seine organischen Strukturen oder Oberflächen würdigen.

In welchen Schritten verlief Ihre autodidaktische Ausbildung zum „Botanical Artist“? 


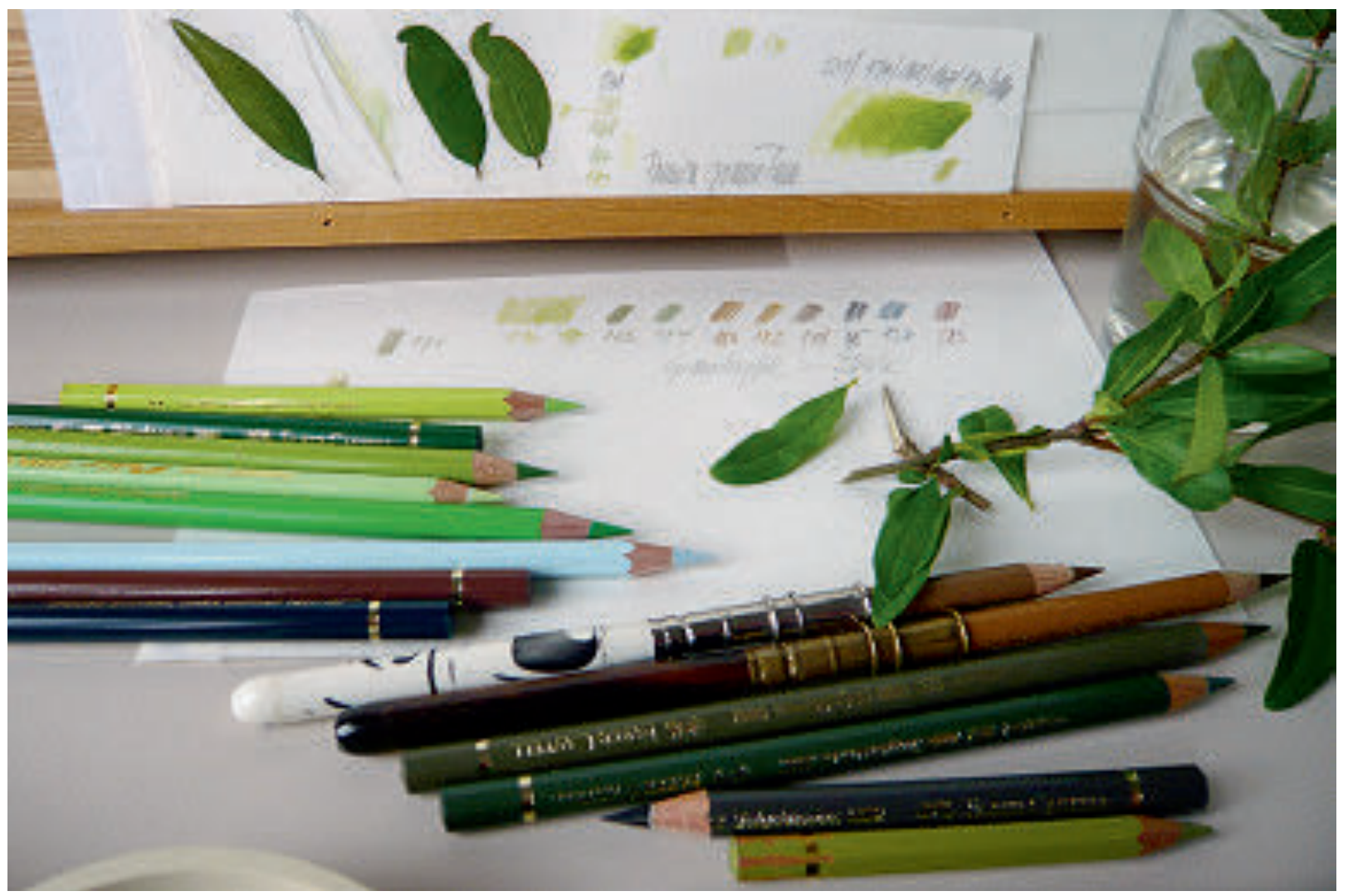

Wir in Deutschland haben eine lange Tradition, man denke an Dürer, Ehret, die Gebrüder Bauer oder natürlich an Maria Sibylla Merian. Leider führt im Gegensatz zu den angelsächsischen Ländern, die auch heute eine sehr lebendige Szene haben, botanische Kunst bei uns zu Unrecht ein Nischendasein. Ich musste lange nach Ausbildungsmöglichkeiten suchen und hatte dann das Glück, durch einen Lehrer der Fachhochschule Krefeld mit privaten Studien unterstützt zu werden. Als Mitglied der American Society of Botanical Artists konnte ich auf deren jährlichen Konferenzen Kurse und Vorträge besuchen. Hier kann man neue Techniken unter fachkundiger Anleitung ausprobieren, erlernen und sich mit all den anderen Teilnehmern austauschen. Und natürlich sollte man möglichst viele Ausstellungen besuchen, um das Auge zu schulen.

\section{Beschreiben Sie bitte den langen Weg vom ersten Kontakt mit Ihrem Wunschobjekt}

Abb. 1: Gute Stifte sind unverzichtbar. und der endgültigen, für Sie perfekten Zeichnung.

Die perfekte Zeichnung gibt es (für mich) nicht. Am längsten hat wohl die Suche nach Gelben Beten gedauert. Ich musste sie fast zehn Jahre suchen, um sie endlich zu Papier zu bringen. Und am Beispiel der 'Blauhilde' lässt sich der manchmal recht lange Weg gut beschreiben. Ich sah die reifen Bohnen im Biologischen Zentrum in Lüdinghausen, einer Umwelteinrichtung, im Beet der Pflanzen aus der Neuen Welt. Als ich zu Hause ankam, waren die Blätter allerdings schon verwelkt. So habe ich in dem Jahr nur die Bohnen gezeichnet. Für solche Situationen habe ich ein kleines Beet in meinem Garten, geschützt gegen Schnecken und Kaninchen, um den ganzen Wachstumszyklus einer Pflanze verfolgen zu können. Also legte ich im nächsten Jahr Samen aus. Leider war der Bohne etwas nicht recht, sie wuchs nicht und das Ergebnis lud nicht zum Zeichnen ein. Im Jahr darauf startete ich einen neuen Versuch. Diesmal hatte ich alles: schöne Blätter und Blüten als Ergänzung zu den Bohnenhülsen und Samen. Wir kennen auch alle den Gra- 


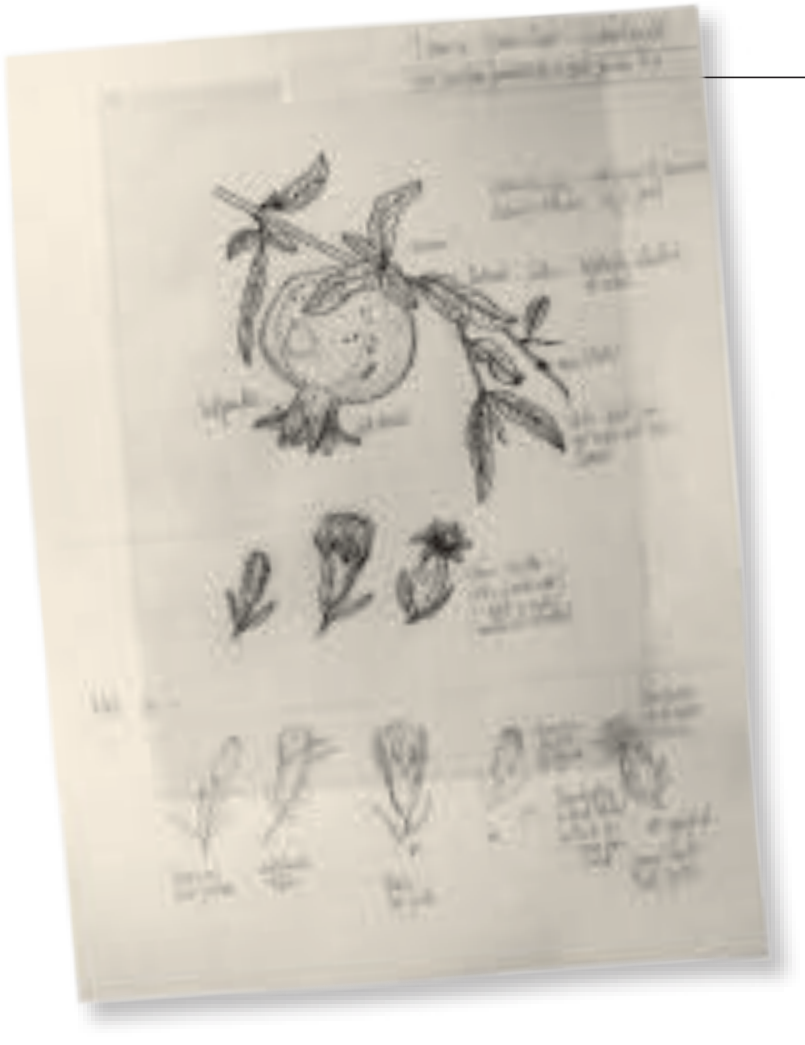

natapfel aus dem Supermarkt, aber wie sehen Zweige, Blätter, Blüten oder Samen aus? Das bestimmt den Weg zu meinem Wunschobjekt.

Vermutlich hat Ihre Leidenschaft für das Zeichnen botanischer Objekte auch Ihr Leben, Ihre Persönlichkeit sowie das Verhältnis zur Natur geprägt bzw. beeinflusst, woraus sich dann auch Ihr ganz persönlicher Stil entwickelt hat. Wie sieht diese Wechselwirkung in der Realität aus?

Gemüse und Obst sind Körper mit glatten, rauen, samtenen oder pergamentartigen Oberflächen. Mit meinen Farbstiften lässt sich die Körperlichkeit der Früchte und Oberflächenbeschaffenheit sehr gut darstellen. Man muss die pergamentartige Beschaffenheit der Zwiebelschale ebenso wie die samtige Oberfläche einer Dicken Bohne zu spüren glauben.

Die Oberfläche etwa einer Tomate ist nie einfarbig rot oder die einer Bohne einfarbig grün. Kleine Unebenheiten und Poren der $\mathrm{Au}-$ ßenschale werfen Schatten; Spiegelungen von Licht oder eines blauen Himmels bedürfen vieler übereinanderliegender hauchzarter Farbschichten, bis die Farbtöne perfekt getroffen sind und Tiefe entsteht. Das versuche ich mit meinen Arbeiten einzufangen. Zuerst mache ich eine möglichst genaue Skizze. Ich zeichne

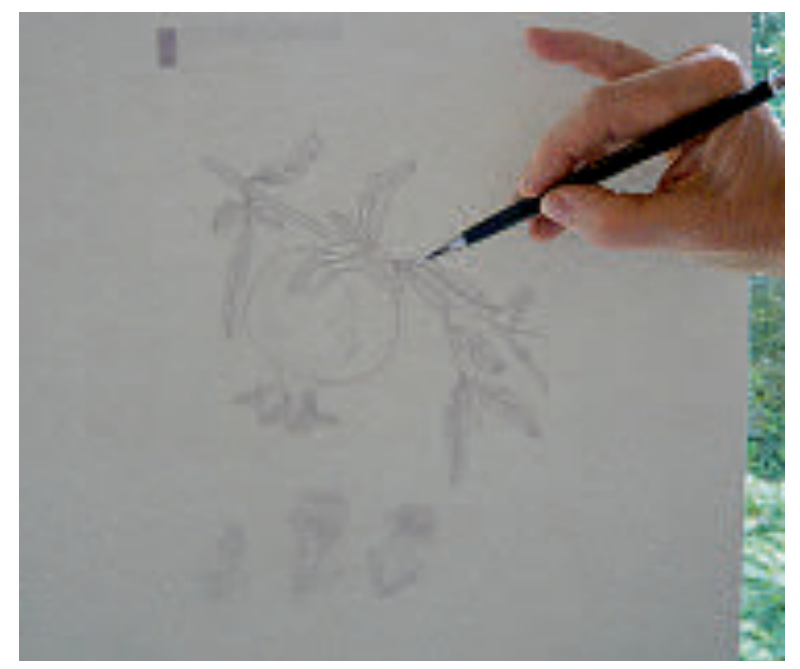

auf Aquarellpapier mit satinierter Oberfläche und muss die Skizze mittels Kopierpapier, Leuchtkasten oder Fenster als Ersatz für den Leuchtkasten darauf übertragen. Ich zeichne die Skizze auf Folie durch und klebe sie an mein Fenster und darüber mein Zeichenpapier. Mit ganz feinen Bleistiftstrichen zeichne ich nun die wichtigsten Linien nach. Danach arbeite ich endlich mit meinen vielen farbigen Stiften an meinem Zeichenbrett weiter. Meine Beziehung zur Natur wurde durch das ständige Beobachten der Pflanzen immer enger. Durch die Kombination von genauer Betrachtung und der haptischen Wahrnehmung der Oberflächen erschloss sich mir eine ganz neue Welt. Zum Schluss würde ich noch gerne auf eine weltweite Ausstellungsreihe mit botanischer Kunst im Jahr 2018 hinweisen. Künstler aus 14 verschiedenen Ländern schließen sich zusammen, porträtieren Pflanzen ihrer Heimat und lenken so den Blick auf die unendliche Vielfalt der Flora unserer Erde. Der deutsche Beitrag wird in Thüngersheim im Forum Botanische Kunst gezeigt werden.

Weitere Informationen unter www.botanische-kunst.de.

Abb. 2 (links): Skizzen des Granatapfels nach Originalvorlage.

Abb. 3 (rechts): Durchzeichnen der Skizze.

Abb. 4 (Seite 127): Die fertige Granatapfel-Zeichnung. 


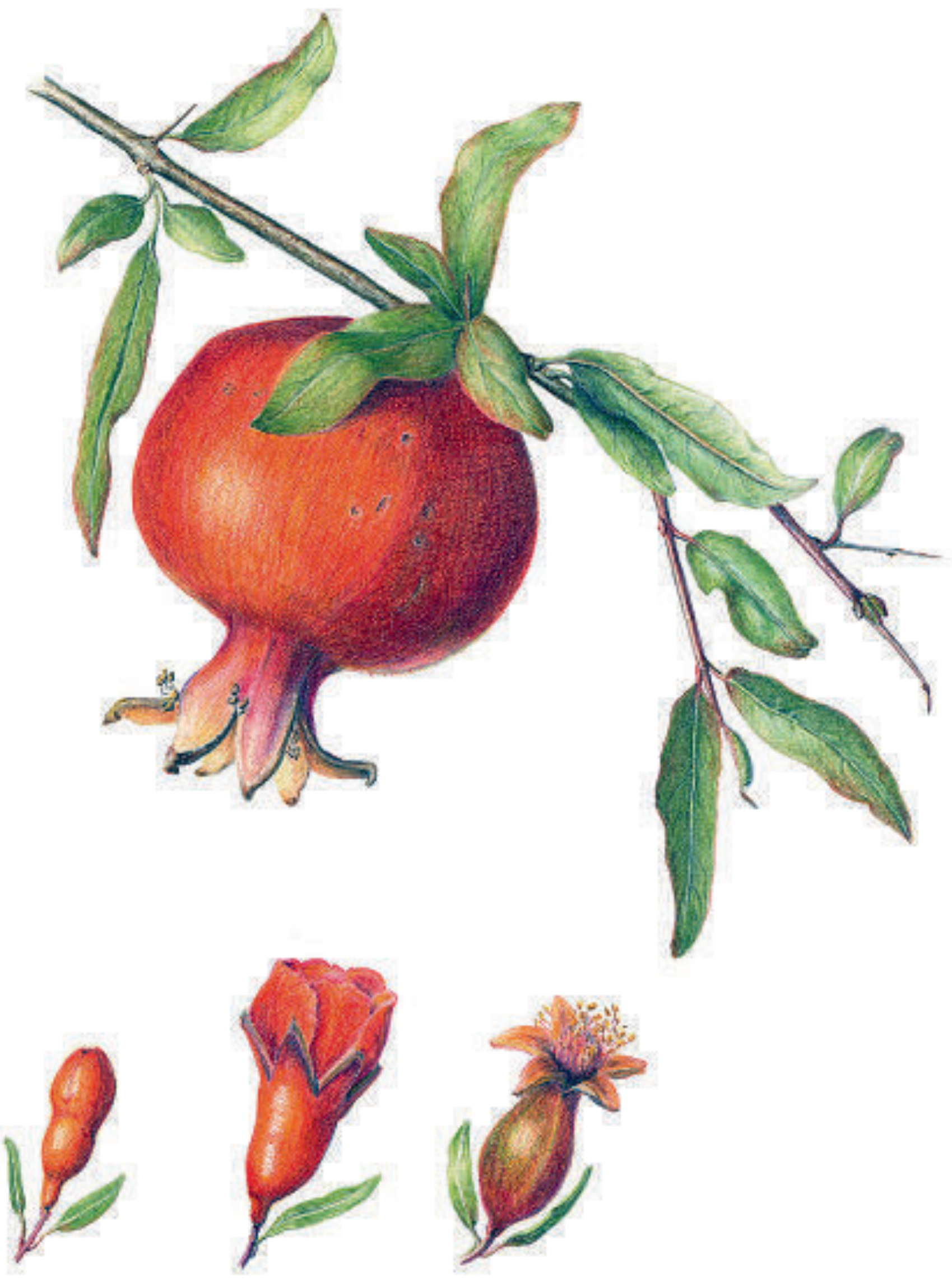

